\title{
Direct visualization of cAMP signaling in primary cilia reveals up-regulation of ciliary GPCR activity following Hedgehog activation
}

\author{
Jason Y. Jiang ${ }^{a, b}$, Jeffrey L. Falcone ${ }^{a}$, Silvana Curci ${ }^{a, b}$, and Aldebaran M. Hofer ${ }^{a, b, 1}$ \\ aVA Boston Healthcare System, Brigham \& Women's Hospital and Harvard Medical School, West Roxbury, MA 02132; and bepartment of Surgery, \\ Brigham \& Women's Hospital and Harvard Medical School, West Roxbury, MA 02132
}

Edited by David E. Clapham, HHMI, Ashburn, VA, and approved May 7, 2019 (received for review November 18, 2018)

\begin{abstract}
The primary cilium permits compartmentalization of specific signaling pathways, including elements of the Hedgehog (Hh) pathway. Hh transcriptional activity is thought to be negatively regulated by constitutively high ciliary CAMP maintained by the Go(s)-coupled GPCR, GPR161. However, cilia also sequester many other Go(s)-coupled GPCRs with unknown potential to regulate $\mathrm{Hh}$. Here we used biosensors optimized for ciliary CAMP and strategies to isolate signals in the cilium from the cell body and neighboring cells. We found that ciliary CAMP was not elevated relative to cellular CAMP, inconsistent with constitutive CAMP production. G $\alpha(s)$-coupled GPCRs (e.g., the $5-\mathrm{HT}_{6}$ serotonin and D1R dopamine receptor) had reduced ability to generate CAMP upon trafficking to the ciliary membrane. However, activation of the Hh pathway restored or amplified GPCR function to permit cAMP elevation selectively in the cilium. Hh therefore enables its own local GPCR-dependent CAMP regulatory circuit. Considering that GPCRs comprise much of the druggable genome, these data suggest alternative strategies to modify Hh signaling.
\end{abstract}

FRET biosensors | calcium | cyclic AMP | primary cilium

ompartmentalization of signal transduction is a recurring theme throughout biological systems. Localized signaling promotes energetic efficiency, maintains fidelity, and allows a limited repertoire of second messengers (e.g., cAMP and $\mathrm{Ca}^{2+}$ ) to be repurposed to control multiple (sometimes conflicting) biological outcomes (1). The primary cilium is an organelle that has been described as a signaling hub because it concentrates a large number of specialized signaling components within its tiny volume. In particular, primary cilia host diverse GPCRs connected to cAMP signaling $(2,3)$. Ciliary GPCRs linked to $\mathrm{G} \alpha_{\mathrm{s}}$ to catalyze the formation of cAMP include 5-HT 6 , V2R, EP4, D1R, and TGR5, while SSTR3, NPY2R, KISS1R, MC4R, and MCHR1 are among the ciliary receptors that inhibit adenylyl cyclase through $\mathrm{G} \alpha_{\mathrm{i}}(2,4,5)$. This ciliary localization is sometimes quite exclusive. For example, the $5-\mathrm{HT}_{6}$ receptor, a neuronal GPCR with apparent functions in cognition and memory consolidation (6), can be highly restricted to the cilium (7-9). G $\alpha_{\mathrm{s}}$ (GNAS), adenylyl cyclases (ADCY3, 5, 6 , and 10), phosphodiesterases (PDE4C), PKA holoenzyme, and numerous PKA substrates are also established residents of the primary cilium $(10,11) ;(12-16)$. Taken together, an extremely attractive model can be envisaged in which 2 cAMP signaling compartments are maintained concurrently in cilium and cell, allowing the same soluble messenger (cAMP) to independently control distinct biological functions. However, the nature of the ciliary signaling microdomain and direct proof that GPCRs function in their usual way have been little explored.

Interest in ciliary cAMP signaling has expanded greatly following the discovery that cAMP-dependent protein kinase A (PKA) is among the kinases regulating the ciliary transcription factors that mediate canonical Hedgehog (Hh) signaling (i.e., through phosphorylation of Gli transcription factors) $(17,18)$. The prevailing view is that a high resting [cAMP $]_{\text {cilia }}$ (relative to the cytosol) is maintained in the absence of Hh ligand, leading to PKA-dependent modulation of Gli $(15,17,19)$. GPR161, an orphan receptor that exits the cilium upon $\mathrm{Hh}$ activation, is believed to be constitutively active and has been proposed to supply the cAMP needed for suppression of $\mathrm{Hh}$ signaling under resting conditions $(17,18)$. Meanwhile, smoothened (Smo), a GPCR-like protein central to activation of the $\mathrm{Hh}$ signaling, enters the cilium following Hh activation (20). Smo is thought to work through $G \alpha_{i}$ to decrease cAMP signaling in the cilium and is therefore permissive for Gli engagement.

From this model, it is apparent that additional ciliary GPCRs might impinge on Gli proteins and other ciliary targets. However, the cAMP signaling properties of the cilium have mostly been inferred, e.g., from cAMP measured in whole cells (17, 21-23), and in only a few cases has the second messenger been measured directly in this organelle $(19,24,25)$. Applying in vitro calibration curves to fluorescence levels measured from a cilium-targeted cAMP reporter, Moore et al. (19) estimated resting [cAMP $]_{\text {cilia }}$ in mIMCD3 cells to be $\sim 4.5 \mu \mathrm{M}$, compared with $<1 \mu \mathrm{M}$ in the cytosol. This [cAMP] would be fully able to persistently activate ciliary cAMP effectors such as PKA and Epac and thus maintain repression of $\mathrm{Hh}$.

Here we revisited the question of whether GPCR-dependent cAMP signals can be generated independently within the cilium. Using Förster resonance energy transfer (FRET)-based reporters optimized for $[\mathrm{cAMP}]_{\text {cilia }}$ measurements, we focused on 5 GPCRs previously reported to reside in cilia, EP4, V2R, D1R, SSTR3, and $5-\mathrm{HT}_{6}$. The cAMP-generating activity of certain GPCRs was significantly up-regulated by $\mathrm{Hh}$ signaling. In conflict with prior reports (19), we also did not find evidence for elevated basal [cAMP] in the cilioplasm of 3 different cell types. Our direct organelle measurements challenge some of the assumptions about the nature of cAMP signaling in the ciliary space and indicate possible roles for GPCRs other than GPR161 in Hh repression.

\section{Significance}

The primary cilium, a small organelle essential to normal development, has recently been thrust into the limelight owing to its involvement in adult disease states such as cancer, obesity, neurodegeneration, and polycystic kidney disease. Here we developed optical approaches to interrogate the function of receptors that work through CAMP-PKA signaling within the primary cilium of live cells. Our data provide a key piece of the puzzle concerning the way in which localized CAMP signals regulate the Hedgehog pathway, another ciliary signaling system with emerging roles in multiple disease processes.

Author contributions: J.Y.J., J.L.F., S.C., and A.M.H. designed research; J.Y.J. and J.L.F. performed research; J.Y.J. and J.L.F. contributed new reagents/analytic tools; J.Y.J., J.L.F., S.C., and A.M.H. analyzed data; and J.Y.J., J.L.F., S.C., and A.M.H. wrote the paper The authors declare no conflict of interest. This article is a PNAS Direct Submission. Published under the PNAS license.

${ }^{1}$ To whom correspondence may be addressed. Email: ahofer@rics.bwh.harvard.edu.

This article contains supporting information online at www.pnas.org/lookup/suppl/doi:10 1073/pnas.1819730116/-/DCSupplemental.

Published online May 29, 2019. 


\section{Results}

Imaging cAMP in the primary cilium of live cells using fluorescent biosensors presents several unique challenges. First, cilia are small and slender, protruding 2-10 $\mu \mathrm{m}$ above the surface of the cell; second, while nonmotile, they are subject to movement due to bulk flow in the bathing solution, and third, bona fide ciliary signals can be difficult to separate optically from background fluorescence emanating from the cell body. While several cilium-targeted cAMP reporters have been described previously $(19,24,25)$, we sought to generate ratiometric biosensors with improved brightness and targeting fidelity. After testing numerous designs, we found the best targeting, brightness, and sensitivity were offered by the fourth-generation Epac-based probes from the laboratory of Kees Jalink (EpacH188 and a higher-affinity version, EpacH187) (26) combined with Arl13b, a protein highly localized to cilia (27) (Fig. 1A). Sensors incorporating the 5-HT 6 receptor gave comparably good localization as Arl13b-coupled probes; however, as discussed below, $5-\mathrm{HT}_{6}$ receptors not localized to cilia caused elevation of cellular cAMP due to the well-known constitutive activity of this GPCR (28).

When expressed in mIMCD3 cells, a cell type widely used in studies of ciliary biology that readily forms well-characterized cilia in culture $(4,10,29)$, the fluorescence of Arl13b-H188 or Arl13b-H187 was largely confined to the primary cilium (Fig. $1 A$; see also SI Appendix, Fig. S1). As often occurs with expression of cilium-targeted proteins, we did note that transfection with the sensor caused an approximate doubling in ciliary length as measured
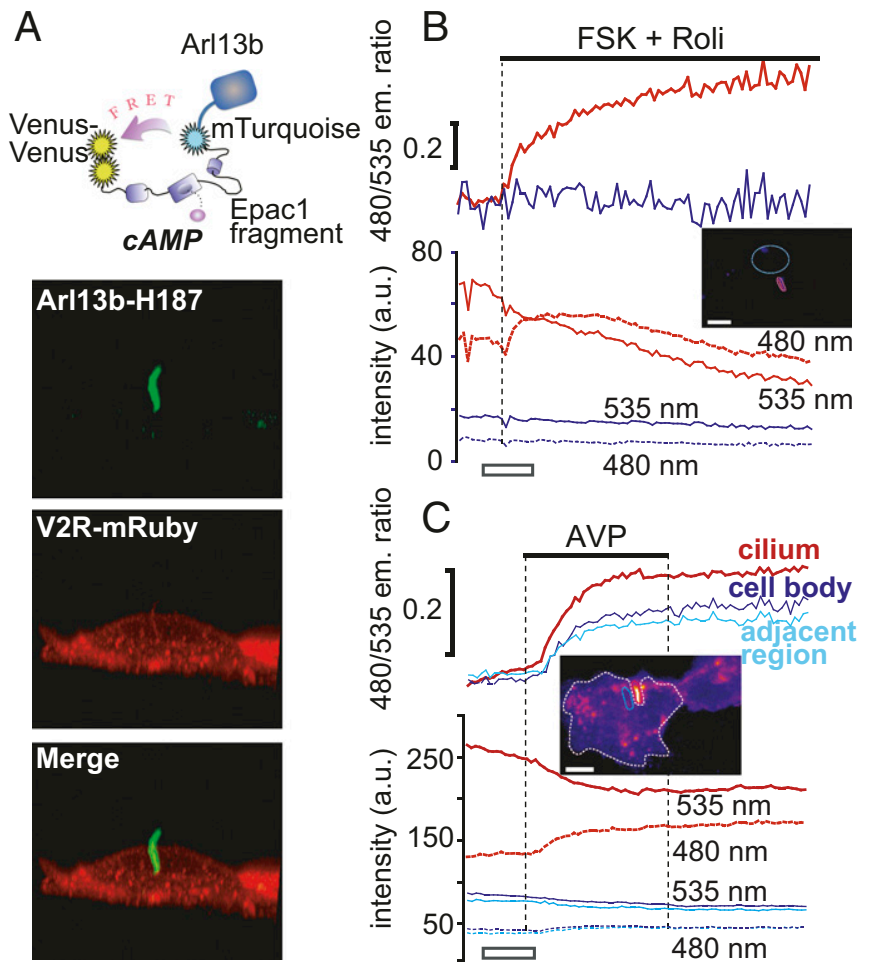

Fig. 1. Validation of ciliary CAMP sensors. (A) Three-dimensional reconstruction of Airyscan images of live mIMCD3 cells cotransfected with the FRET sensor Arl13b-Epac-H187 (green) and V2R-mRuby (red). Inset: model of construct design for Arl13b-H187/H188. (B) Well-targeted Arl13b-H187 reports CAMP changes in the primary cilium of mIMCD3 cells in response to $50 \mu \mathrm{M}$ forskolin (FSK) $+20 \mu \mathrm{M}$ rolipram (Roli) (typical of $n=4$ experiments, 4 primary cilia). (C) mIMCD3 cells transiently expressing V2R-mRuby and Arl13b-H187 responded robustly to $50 \mathrm{pM}$ AVP in both cilia and cytosol (typical of $n=6$ experiments, 14 primary cilia). Blue traces, cell body; red traces, primary cilium. Time bar: $3 \mathrm{~min}$. using an unbiased custom script (SI Appendix, Fig. S2; see SI Appendix, Materials and Methods for details). FRET emission wavelengths were sampled using a high numerical aperture (N.A.) (1.45) oil immersion objective. When a nearby region was recorded in the cell body, the fluorescence was typically less than $20 \%$ of that of the cilium. In many cells (Fig. $1 B$ ), cytosol-derived background fluorescence was so low that it did not contribute to the ciliary signal whatsoever, showing that it is possible to exclusively monitor ciliary [cAMP] with this probe. However, in other cells (e.g., V2R-transfected mIMCD3 cells stimulated with $50 \mathrm{pM}$ arginine vasopressin (AVP) depicted in Fig. 1C), it was possible to also resolve signaling events in the cytosol after collecting fluorescence over the entire cell body (minus the cilium). It should be emphasized that the large reciprocal changes in intensity in the 480 and $535 \mathrm{~nm}$ emission (em.) wavelengths in the ciliary region still far exceeded the magnitude of excursions monitored in a nearby cytosolic region. This indicates the signal collected over the ciliary region is minimally contaminated by nontargeted reporter retained in the cell.

Taking advantage of the ability to monitor cell and cilium simultaneously, we noted that the resting FRET ratio in the cilium did not, on average, appear to be consistently different from that measured in the cytosol, suggesting no gradient in resting ciliary [cAMP]. However, targeted biosensors can sometimes exhibit dramatically different behaviors depending on their cellular environment; thus, calibrations are needed to confirm that these ratios reflect true cAMP levels. Therefore, we performed an in situ calibration of the cAMP reporters, capitalizing on the finding that in $\mathrm{mIMCD} 3$ cells digitonin can selectively permeabilize the plasma membrane but not the ciliary membrane, which is reported to be deficient in cholesterol $(30,31)$. Both ciliary and nontargeted Arl13bH188/H187 were retained completely after semipermeabilization and proved equivalently responsive to stepwise increases in cAMP $(0 \mu \mathrm{M} \rightarrow 5 \mu \mathrm{M} \rightarrow 50 \mu \mathrm{M})$ in the bathing solution. With both sensors we did not observe any difference between $[\mathrm{cAMP}]_{\text {cilia }}$ and [cAMP $]_{\text {cytosol }}$ (Fig. $2 A$ and $B$ ). Overnight treatment of cells with smoothened agonist (SAG) or Shh ligand to activate the Hedgehog signaling pathway did not significantly alter this pattern (SI Appendix, Fig. S3 $A$ and $B$ ).

Our initial calibrations indicated that basal [cAMP] in both compartments was maintained at submicromolar levels. We performed more detailed in situ calibrations with the higher-affinity probe, Arl13b-H187, using a lower range of cAMP concentrations in mIMCD3 cells (SI Appendix, Fig. S3 $C-E$ ). We determined that the targeted biosensor can report changes in [cAMP] below $500 \mathrm{nM}$ and estimated that the average resting [cAMP] in mIMCD3 cells was $0.72 \pm 0.27 \mu \mathrm{M}$ in cilia and $0.90 \pm 0.72 \mu \mathrm{M}$ in the cytosol [not significant (N.S.); $P=0.817$ ]. Ciliary and cytosolic [cAMP] were estimated in 2 other cell lines, MEF and 3T3 cells, with similar results (in MEFs: cAMP $=0.21 \pm 0.30 \mu \mathrm{M}$ in cilia versus $0.72 \pm 0.29 \mathrm{nM}$ in the cell body, N.S.; $P=0.264$; in 3T3: $0.75 \pm 0.18 \mu \mathrm{M}$ in cilia versus $0.19 \pm 0.43 \mu \mathrm{M}$ in the cell body, N.S.; $P=0.267$; see SI Appendix for details).

We next selected 5 receptor systems purported to reside in the cilium (EP4, V2R, 5- $\mathrm{HT}_{6}, \mathrm{D} 1 \mathrm{R}$, and SSTR3) to evaluate the characteristics of agonist-stimulated ciliary cAMP signaling events. A previous immunofluorescence staining study reported that EP4 prostaglandin receptors are localized to primary cilia in mIMCD3 cells (4). We therefore transfected mIMCD3 cells with a functional mCherrytagged EP4 receptor (SI Appendix, Fig. S4). We noted only modest levels of mCherry fluorescence in the primary cilium but did observe bright labeling in the cell body (Fig. $3 A$ ). This result raises the possibility that the mCherry tag altered the localization of EP4. Using Epac-H188, we observed robust and reversible FRET ratio changes in response to $100 \mathrm{nM}$ PGE1 (Fig. $3 B$ ) in the cytosol of virtually all mIMCD3 cells examined. Similar signals were recorded in primary cilia using the Arl13b-targeted reporter. Fig. $3 C$ shows that the perfect targeting of the sensor allowed exclusive monitoring 

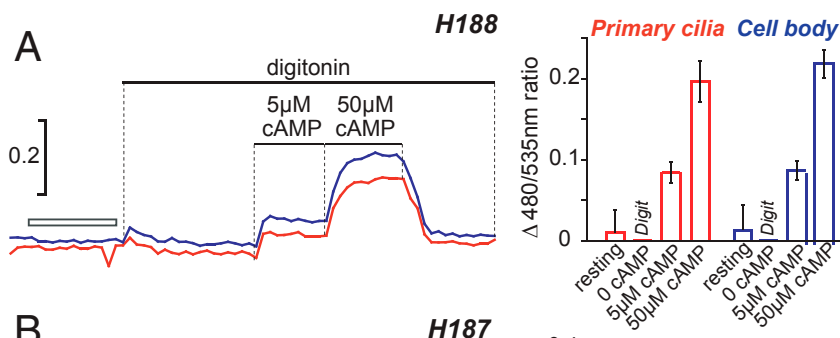

B

$H 187$
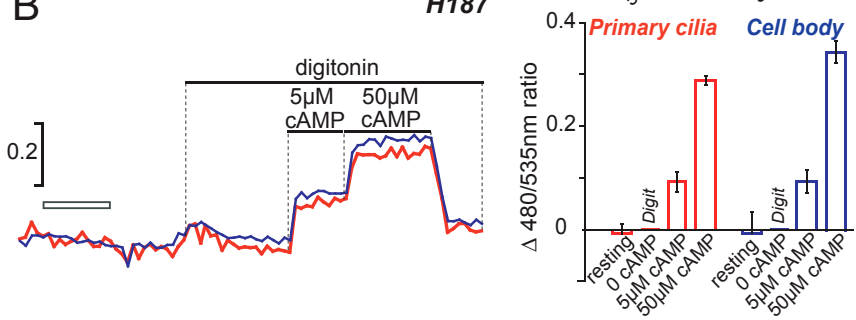

Fig. 2. Resting ciliary CAMP is not elevated relative to the cytosol. ( $A$ ) Ciliary CAMP was calibrated using loosely targeted Arl13b-Epac-H188 $\left(K_{\mathrm{d}}\right.$ for CAMP $\sim 10 \mu \mathrm{M})$ in digitonin-permeabilized mIMCD3 cells and serially increasing CAMP buffer concentrations. The resulting response curves were not significantly different in cilia versus cell body (typical of $n=4$ experiments, 7 primary cilia/cells). (B) Calibration of Arl13b-Epac-H187 was performed as above $(n=6$ experiments with 6 primary cilia/cells, $P=0.9741$ ). See also SI Appendix, Fig. S3 for additional details. Blue traces, cell body; red traces, cilia. Time bar: $3 \mathrm{~min}$ The $y$ axis depicts the 480/535 FRET ratio.

of ciliary cAMP. Assuming that the endogenous EP4 receptor is localized to cilia in our cells, this signal could be derived either from within the cilium through activation of ciliary EP4 receptors, via communication with cAMP synthesized in the cell body, or with some combination thereof.

The V2R vasopressin receptor was reported to reside on the cilium of renal cells (32). As mIMCD3 cells proved to have no endogenous expression of functional V2R, we transfected cells with mRuby2-, mScarlet-, or EGFP-tagged versions of the receptor. Analysis of the 3-dimensional distribution of V2R in these cells showed that the vast majority of the GPCR was in the plasma membrane, with a relatively low density of $\mathrm{V} 2 \mathrm{R}$ in the cilium (Fig. 3D, arrow; see SI Appendix, Fig. S5 for quantification). Expression of these constructs rendered the cells uniformly sensitive to low physiological concentrations of AVP (Fig. $3 E$ ). Interestingly, in contrast to PGE1, the response to AVP was only rarely reversible after agonist washout, perhaps reflecting the continued activation of internalized V2R from an endosomal compartment $(33,34)$. Persistent cAMP elevation was also observed in the primary cilium using targeted sensors; Fig. $3 F$ depicts an experiment in which it was possible to monitor 2 cell bodies and their associated cilia in the same microscope field. The ciliary and cellular signals mirror one another. We conclude that the cAMP signal measured in the cilium after AVP stimulation is generated principally in the cytosol and, as expected (8), communicated freely to the cilium by diffusion.

We then examined the effects of stimulating the ciliary $5-\mathrm{HT}_{6}$ receptor. As mIMCD3 cells do not express native $5-\mathrm{HT}_{6}$, mCherry-5- $\mathrm{HT}_{6}$ was coexpressed with targeted or nontargeted cAMP reporters. Fig. $3 G$ illustrates that $5-\mathrm{HT}_{6}$ receptors can segregate exclusively to the primary cilium, although cells harboring receptors in both the cilia and cell body were also noted (e.g., see SI Appendix, Fig. S6). These latter cells could be reliably stimulated with serotonin (5-HT) to yield increased FRET ratios (Fig. $3 H$ ). We expected that we should be able to directly visualize agonist-dependent signals in ciliated cells in which the GPCR was exclusively localized to the cilium. However, to our surprise, we were never able to record a cAMP change in either the cilium or

\section{A}
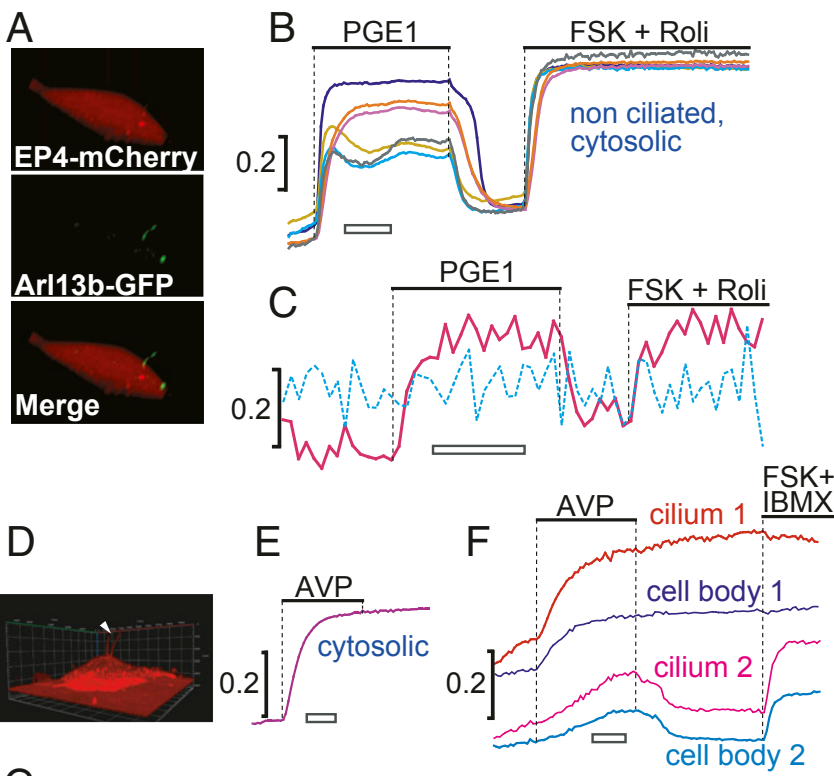

G

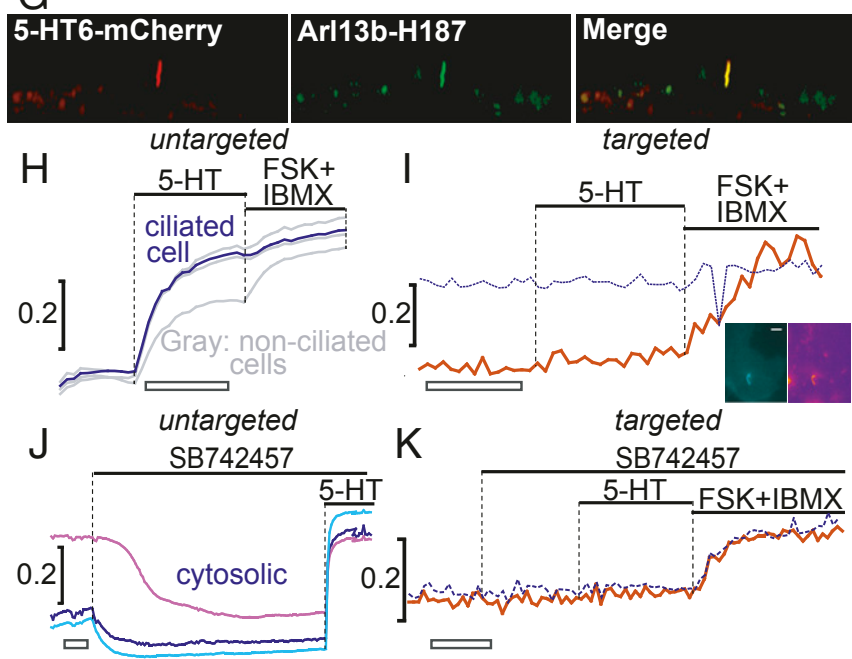

Fig. 3. Evaluation of putative ciliary GPCRs: EP4, V2R, and 5-HT6. (A) Threedimensional Airyscan images of a ciliated mIMCD3 cell expressing EP4-mCherry (red) and Arl13b-GFP (green). ( $B$ ) FRET ratio changes in multiple mIMCD3 cells stimulated with 100 nM PGE1 as measured by nontargeted Epac-H187, followed by $50 \mu \mathrm{M}$ forskolin $+20 \mu \mathrm{M}$ rolipram (typical of 4 experiments, 38 cells). (C) Typical ciliary CAMP in response to PGE1 as measured by Arl13b-Epac-H187 (17 experiments, positive responses in 13 of 20 cilia). (D) Three-dimensional Airyscan image of a mIMCD3 cell expressing V2R-mRuby2 (white arrow indicates cilia). (E) Persistent CAMP signal after treatment with 50 pM AVP in mIMCD3 expressing V2R-mRuby2 and cytosolic Epac-H187 (typical of 4 experiments, 38 cells). (F) AVP produced similar CAMP responses in cilia and cytosol as measured by Arl13bEpac-H187 (typical of 6 experiments, 14 cilia). (G) Three-dimensional Airyscan images of a ciliated cell expressing $5-\mathrm{HT}_{6}$-mCherry (red) and Arl13b-Epac-H187 (green). $(H)$ mIMCD3 cells expressing poorly targeted $5-\mathrm{HT}_{6}$ and untargeted Epac- $\mathrm{H} 187$ responded robustly to $10 \mu \mathrm{M} 5-\mathrm{HT}$ in ciliated and nonciliated cells (6 experiments, $9 / 25$ cells ciliated). (I) When $5-\mathrm{HT}_{6}$ was strictly confined to the ciliary membrane (indicated by mCherry fluorescence), treatment with 5-HT caused no change in CAMP ( 6 experiments, 6 cilia). In this experiment, there was insufficient FRET sensor in the cytosol to report cytosolic CAMP changes, explaining the lack of response to $50 \mu \mathrm{M}$ forskolin and $1 \mathrm{mM}$ IBMX (3-isobutyl-1-methylxanthine: noisy blue dotted trace). ( () When $5-\mathrm{HT}_{6}$ was localized to the cell membrane, the inverse agonist, SB742457 $(1 \mu \mathrm{M})$, caused a reduction in [CAMP] $]_{\text {cytosol }}$ levels, and 5 - $\mathrm{HT}$ reversed the effect (typical of 3 experiments, 16 cells). ( $K$ ) When $5-\mathrm{HT}_{6}$ was localized strictly to the ciliary membrane, SB742457 had no effect on CAMP levels in the cell body or cilia (typical of 7 experiments, 11 cilia). Blue traces, cell body; red traces, cilia. Time bar: 3 min unless otherwise noted; the $y$ axis depicts the 480/535 nm FRET ratio. 
cytosol (Fig. 3I) under these circumstances. We observed that receptor expression in nonciliated cells results in constitutive elevation of cellular cAMP, as reported previously (6). The constitutive activity of the receptor was unmasked using the inverse agonist SB742457, causing variable reduction in the baseline ratios (Fig. 3J), and effectively restored using the native agonist, 5-HT (Fig. 3J). Similarly, when 5- $\mathrm{HT}_{6}$ was perfectly localized, we failed to observe any effect of the inverse agonist on ciliary cAMP (Fig. $3 K$ ), suggesting that the usual constitutive activity was eliminated once the GPCR was in the ciliary membrane. Note that these experiments were conducted in the presence of gap junction inhibitors to prevent communication of cAMP between ciliated and nonciliated GPCR-expressing cells (35).

We next employed a permeabilized cell model with the aim of isolating the ciliary response from that in the rest of the cell. We initially focused on the $5-\mathrm{HT}_{6}$ receptor because we could confirm definitively its ciliary localization. Cells were treated briefly with a pulse of digitonin in an "intracellular-like" buffer supplemented with ATP and GTP and free $\left[\mathrm{Ca}^{2+}\right]$ clamped to $180 \mathrm{nM}$ (Fig. 4A). We reasoned that the digitonin would render the cell body incapable of accumulating cAMP following agonist stimulation, while the sensor in the restricted microdomain of the primary cilium should be able to register local cAMP production. A pulse of cAMP was given at the end of every experiment to confirm permeabilization of the plasma membrane. The response to 5-HT in the cell body was completely eliminated following permeabilization. However, again, no response to 5-HT in the cilia was ever observed in mIMCD3 cells under these conditions, even though the organelle clearly harbored the GPCR (Fig. 4A). We also saw no effect of the inverse agonist (Fig. $4 B$ ) under these conditions. Experiments aimed at assessing the activity of EP4 and V2R in permeabilized cells gave comparable results (SI Appendix, Fig. S7).

An obvious explanation for the lack of effect in the preceding experiments was that cAMP did not accumulate sufficiently to be captured by the reporter. However, when the same experiment was repeated using forskolin to directly activate endogenous ACs
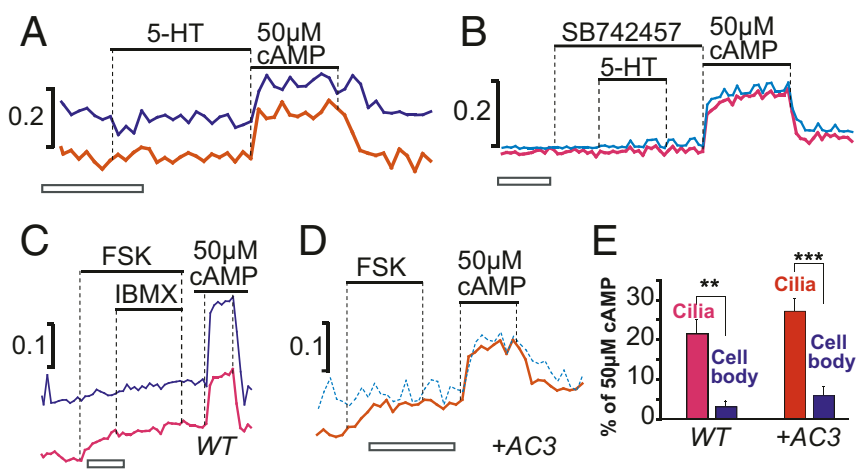

Fig. 4. CAMP signaling in cilia of digitonin-permeabilized cells. All experiments were performed in intracellular-like buffer supplemented with ATP and GTP. (A) mIMCD3 cells expressing $5-\mathrm{HT}_{6}-\mathrm{mCherry}$ and Arl13b- $\mathrm{H} 187$ localized to both the cell and cilium did not respond to $10 \mu \mathrm{M} 5-\mathrm{HT}$ in the cell body or in the cilium after $2 \mathrm{~min}$ treatment with $20 \mu \mathrm{g} / \mathrm{mL}$ digitonin (4 experiments, 5 cilia). (B) Similarly, responsiveness to the inverse agonist, $1 \mu \mathrm{M}$ SB742457, and subsequent $5-\mathrm{HT}_{6}$ rebound response were lost in both the cell and cilium after 2 min treatment with $20 \mu \mathrm{g} / \mathrm{mL}$ digitonin (3 experiments, 3 cilia). (C) Digitonin permeabilization ablated the $50 \mu \mathrm{M}$ forskolin-induced CAMP signal in cell bodies, but CAMP increase was detectable in cilia; $1 \mathrm{mM}$ IBMX-induced PDE inhibition did not unmask any signal ( $n=4$ experiments with 4 cilia/7 cells). (D) AC3 cotransfection did not change the forskolin response. $(E)$ Quantification and comparison of CAMP response to forskolin in cell bodies and in cilia; $* * P=$ $0.0002, * \star * P=00019$. Blue traces $=$ cell body, red traces $=$ cilia. Time bars: 3 $\min$. The $y$ axis is the $480 / 535 \mathrm{~nm}$ ratio. (adenylyl cyclases) present in the ciliary membrane, a small but consistent accumulation of cAMP was detected in the microenvironment of the cilium but not in the cell body (Fig. $4 C$ and $E$, left bar graphs). Similar results were obtained in cells expressing AC3 (36) (Fig. $4 D$ and $E$, right bar graphs), which was localized to cilia when expressed in mIMCD3 cells (SI Appendix, Fig. S8). Assuming that permeabilization did not lead to loss of essential components of the GPCR signaling machinery, the small but detectable change following forskolin addition suggests that it should have been possible to resolve GPCR-dependent cAMP signals were they to take place in the cilium. These data also indicate that the ciliary membrane of mIMCD3 cells was left intact following digitonin treatment.

The preceding data leave us with the question as to why GPCRs are not competent to produce cAMP once sequestered in the cilium. Prior studies have cited $\mathrm{Ca}^{2+}$ entry across the ciliary membrane as an important regulator of organelle cAMP owing to the presence of cilia-specific $\mathrm{Ca}^{2+}$-conducting channels (PKD2L1 and PKD2) and $\mathrm{Ca}^{2+}$-regulated $\mathrm{ACs}(\mathrm{AC1}, 8,5$, and 6) $(11,16,23$, $32,37)$. Primary cilia of mIMCD3 cells have been reported to harbor $\mathrm{Ca}^{2+}$-inhibitable AC5 and/or AC6 (10). We therefore considered that the high resting $\left[\mathrm{Ca}^{2+}\right]$ and $\mathrm{Ca}^{2+}$ permeability of the cilium $(38,39)$ could account for the inactivation of ACs coupled to the $5-\mathrm{HT}_{6}$ receptor. To better address this point, we improved the sensor by replacing the Arl13b targeting sequence with a functional $5-\mathrm{HT}_{6}$ receptor to target the cAMP reporterGPCR fusion directly to the cilium (5- $\mathrm{HT}_{6}$-EpacH187). Consistent with the data of Figs. $3 I$ and $4 C$, when targeted exclusively to the cilium, 5-HT did not yield a measurable cAMP signal (Fig. 5A). Exposure of cells to nominally $\mathrm{Ca}^{2+}$ free solutions did not rescue the 5-HT response in cilia (Fig. $5 B$ ), indicating that $\mathrm{Ca}^{2+}$ entry across the ciliary membrane did not suppress the signal. We considered that perhaps expression of $5-\mathrm{HT}_{6}$ altered the ciliary distribution of the $\mathrm{G} \alpha_{\mathrm{s}}$ protein in mIMCD3 cells, but immunostaining experiments suggested this is not the case (SI Appendix, Fig. S9). However, when we tested the effect of 5-HT in cells pretreated with SAG $(250 \mathrm{nM}$ to $1 \mu \mathrm{M})$ to activate Smo receptors (40), we were able to restore the responsiveness of the $5-\mathrm{HT}_{6}$ receptor to agonist (Fig. 5C). Sensitivity to the inverse agonist was also apparent, implying restoration of constitutive activity (Fig. $5 D$ ).

The preceding results left us with the question of whether the unexpected SAG-dependent up-regulation of the $5-\mathrm{HT}_{6}$ receptor expressed in mIMCD3 cells was cell type and/or GPCR specific. Unlike mIMCD3, stimulation of 3T3 cells (Fig. 5E) and MEFs (SI Appendix, Fig. S10A) with 5-HT produced a measurable ratio change that was, however, significantly enhanced following SAG treatment (Fig. 5F and SI Appendix, Fig. S10B). Interestingly, the smoothened receptor antagonist cyclopamine, which might be expected to have the opposite effect of SAG, did not abolish 5-HT-stimulated cAMP signaling in primary cilia of $3 \mathrm{~T} 3$ cells (bar graph, Fig. $5 F$ ).

Using this system, we were also able to evaluate the ability of a ciliary $\mathrm{G} \alpha_{\mathrm{i}}$-coupled GPCR, SSTR3, to reverse serotonin-stimulated cAMP signals in the cilium. Treatment of 3T3 cells coexpressing SSTR3-GFP and 5- $\mathrm{HT}_{6}-\mathrm{H} 187$ with somatostatin reversed 5-HT responses back to baseline within 2 min in the presence and absence of SAG treatment (Fig. $5 G$ and $H$ ), indicating local control of the 5- $\mathrm{HT}_{6}$ function. We then further tested how SAG affected the activity of another ciliary $\mathrm{G}_{\mathrm{s}}$-coupled GPCR, the dopamine D1R receptor (5). D1R-EGFP was coexpressed with 5- $\mathrm{HT}_{6}-\mathrm{H} 187$ in $3 \mathrm{~T} 3$ cells, and cells with fluorescence only in cilia were selected. Ten nanomolar dopamine produced small but measurable cAMP signals in control cells (Fig. 5I), and again, the signal was significantly enhanced after SAG treatment (Fig. 5J; summarized in bar graph).

\section{Discussion}

The GPCR/cAMP pathway is considered one of the cornerstones of the "druggable genome." Thus, the significance of a distinct cAMP signaling circuit in the primary cilium is that it may potentially 

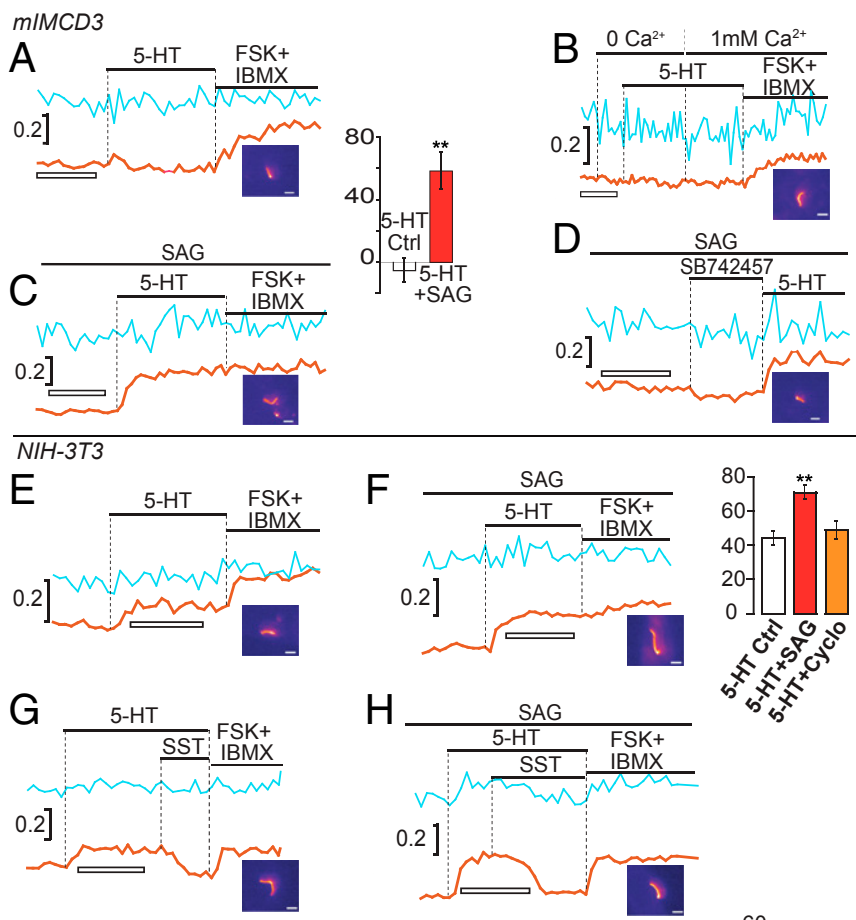

I

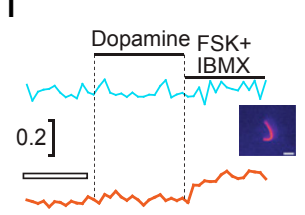

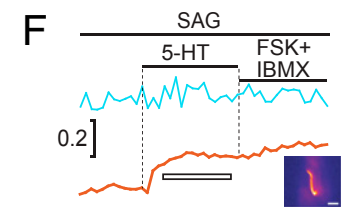

$\mathrm{H}$
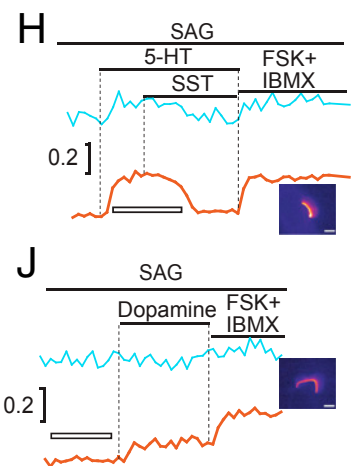
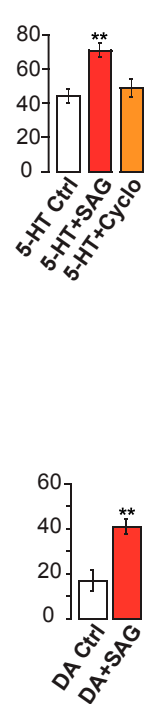

Fig. 5. Hedgehog pathway activation increases CAMP response reported by 5- $\mathrm{HT}_{6}-\mathrm{Epac}-\mathrm{H}_{187}$ to $5-\mathrm{HT}$ and dopamine in primary cilia: $(A)$ Consistent with the data in Figs. $3 /$ and $4 C$, mIMCD3 cells did not respond to $10 \mu \mathrm{M} 5-\mathrm{HT}$ when the sensor/receptor fusion was confined strictly to the ciliary membrane ( 6 experiments, 6 cilia). (B) Attenuating the high $\left[\mathrm{Ca}^{2+}\right]$ in cilia by placing cells in a nominally $\mathrm{Ca}^{2+}$ free solution did not rescue 5-HT-stimulated CAMP signaling (5 experiments, 6 cilia). (C) Following overnight pretreatment with $1 \mu \mathrm{M}$ SAG, 5 out of 6 cilia (6 experiments) responded to $5-\mathrm{HT}$ stimulation ( $* * P=0.000992)$. (D) Pretreatment with $1 \mu \mathrm{M}$ SAG rescued the response to $1 \mu \mathrm{M}$ SB742257 $(-35.5 \pm 11.2 \%$ below baseline relative to the subsequent cAMP increase elicited by $10 \mu \mathrm{M} 5-\mathrm{HT}$; typical of 4 experiments, 4 cilia). (E) A cAMP increase in NIH 3 T3 cilia (typical of 9 experiments, 11 cilia) was caused by $10 \mu \mathrm{M} 5-\mathrm{HT}$. $(F)$ Overnight pretreatment with $1 \mu \mathrm{M}$ SAG significantly increased $5-\mathrm{HT}$ response ( $* * P=0.00034 ; 6$ experiments, 7 cilia). Overnight pretreatment with $10 \mu \mathrm{M}$ cyclopamine (Cyclo) had a negligible effect (8 experiments, 9 cilia, bar graph). ( $G$ and $H$ ) The $100 \mathrm{nM}$ somatostatin (SST) attenuated 5-HT response in cilia harboring SSTR3-GFP with and without SAG pretreatment (G: 5 experiments, 6 cilia; $H: 2$ experiments, 5 cilia). ( $/$ and J) SAG pretreatment increased the $10 \mathrm{nM}$ dopamine (DA) response ( $(: 4$ experiments, 4 cilia, $J: 4$ experiments, 6 cilia, $* * P=0.002476$ ). Blue traces, cell body; red traces, cilia. Time bars: $3 \mathrm{~min}$. The $y$ axis is the 480/ $535 \mathrm{~nm}$ FRET ratio. Bar graphs indicate the 5-HT or DA response as a percentage of the forskolin/IBMX response; error bars represent SEM.

be exploited to intervene on other less druggable pathways localized to this organelle (Wnt, PDGFR $\alpha$, planar cell polarity, etc.). The clearest example to date of a regulatory role for cAMP/PKA in the cilium concerns the Hh pathway $(12,18,21,41)$, aberrations in which are thought to occur in up to $25 \%$ of all cancers, including basal cell carcinoma and medulloblastoma (42, 43). Intraciliary cAMP has also been implicated in other processes, such as the elaboration of dendrites (44), ciliary length control (37), and flow sensing in osteocytes (16), and in the etiology of obesity $(29,45)$. The polycystic kidney disease-related protein PKD2 and other ciliary ion channels are additional potential targets of local cAMP signals

$(32,46)$, although the identity of these conductances and how they are regulated are only starting to be explored $(38,39)$; $(32,47-50)$.

The $5-\mathrm{HT}_{6}$ and $\mathrm{D} 1 \mathrm{R}$ receptors, with their near-exclusive localization to the cilium and well-developed pharmacology $(6,51)$, are potentially poised to fulfill the role of selective activators of ciliary cAMP/PKA targets, including $\mathrm{Hh}$. The neuronal $5-\mathrm{HT}_{6}$ receptor has attracted much interest due to its apparent positive effect on memory consolidation and cognitive function (6). However, a recent series of clinical trials to test the effectiveness of $5-\mathrm{HT}_{6}$ antagonists on Alzheimer's disease yielded disappointing results (52). A more complete appreciation of how and where this GPCR functions could be key to developing more effective drugs.

In this study we overcame some of the technical challenges of measuring dynamic cAMP changes in this small organelle to show that $5-\mathrm{HT}_{6}$ receptors, which were fully functional while in the cell body plasma membrane of ciliated and nonciliated mIMCD3 cells, ceased to generate cAMP upon trafficking to the ciliary membrane. Not only did 5-HT fail to elicit the expected elevation of cAMP, but treatment with an inverse agonist revealed that the constitutive activity of the receptor was also abolished under these conditions. Significantly, this behavior was reversed when cells were treated overnight with the SAG, which causes trafficking of Smo to the cilium to initiate Gli-dependent transcription of Hh target genes (40). In other experiments in 3T3 and MEFs, SAG treatment caused significant up-regulation of $5-\mathrm{HT}_{6}$ and D1R activity. Our data therefore reveal a condition under which 5- $\mathrm{HT}_{6}$ and D1R receptors are predicted to become competent to locally produce cAMP, activate PKA, and provide feedback to repress Gli transcriptional activity.

Our experimental protocol utilized gap junction inhibitors to prevent the transfer of cAMP from cells with functional $5-\mathrm{HT}_{6}$ receptors in the plasma membrane to neighboring ciliated cells with receptor exclusively localized to the primary cilium. Since our data showed that cAMP readily traverses the cytosol to enter the cilium, cAMP communicated from neighboring cells (35) will also enter the cilium to give the false appearance of a ciliumgenerated cAMP change. Attention to this detail in future studies may clarify some of the conflicting results in the field.

The question remains of how and why the activity of the GPCRs examined is regulated in the ciliary milieu. We excluded high intraciliary $\mathrm{Ca}^{2+}(39)$ as the reason for the lack of effect of 5-HT in mIMCD3 cells (Fig. 5B). It is possible that in the absence of $\mathrm{Hh}$ ligand $\mathrm{G} \alpha_{\mathrm{s}}$-coupled GPCRs in the primary cilium experience alternative coupling to other $\mathrm{G} \alpha$ subunits (e.g., $\mathrm{G} \alpha_{\mathrm{i}}$ ). Masyuk et al. (53) provided evidence for such a mechanism using biochemical assays of total cAMP in cholangiocytes expressing the endogenous bile acid receptor, TGR5. Ciliary localization appeared to induce a switch of TGR5 from $\mathrm{G} \alpha_{\mathrm{s}}$-coupled to $\mathrm{G} \alpha_{\mathrm{i}}$ coupled activity. Other explanations for the apparent reduction in GPCR activity include the distinct lipid composition of the ciliary membrane (54), which may not support GPCR function, and the absence of appropriate G $\beta \gamma$ subunits in the cilium (55). SAG treatment might alter the expression, activity, or ciliary accumulation of adenylyl cyclases, cAMP phosphodiesterases, heterotrimeric $G$ proteins, or arrestins. It could also affect the recruitment of other unknown GPCRs that antagonize $\mathrm{G} \alpha(\mathrm{s})$-coupled receptors or lead to changes in the lipid composition of the cilium, ultimately affecting the ability of GPCRs to produce cAMP.

In contrast to prior reports (19), we also did not observe elevated basal [cAMP] in the cilium, nor was baseline ciliary [cAMP] altered by activation of the Hh pathway with SAG or Shh (Fig. 2). These data therefore challenge the assumption that cAMP production occurs constitutively in the ciliary compartment. Our findings do not discount the large body of experimental evidence indicating that ciliary targets are actively regulated by the cAMP/PKA pathway $(12,14,17,18,22,25)$. They do, however, indicate that a more complex cAMP signaling landscape must exist than previously proposed. Most significantly, our data show Smo 
stimulation fosters the development of alternative pathways to local cAMP production. GPCRs such as $5-\mathrm{HT}_{6}$, with its welldeveloped pharmacology and tight localization to cilia, may be adjuncts to therapies that intersect with aberrant Hh signaling.

\section{Materials and Methods}

For full details see SI Appendix, Materials and Methods. Epac1-based CAMP sensors (26), "Epac-H187" and "Epac-H188" (a gift from Dr. Kees Jalink, Netherlands Cancer Institute, Amsterdam, The Netherlands), were targeted to cilia by conjugation with Arl13b or $5-\mathrm{HT}_{6}$. Real-time FRET imaging experiments were performed on a Nikon Eclipse TE2000-U inverted fluorescence microscope equipped with an ORCA ER camera and a 60x Plan Apo TIRF (N.A. = 1.45) oil immersion objective using $440 \mathrm{~nm}$ excitation

1. R. Filadi, T. Pozzan, Generation and functions of second messengers microdomains. Cell Calcium 58, 405-414 (2015).

2. K. I. Hilgendorf, C. T. Johnson, P. K. Jackson, The primary cilium as a cellular receiver: Organizing ciliary GPCR signaling. Curr. Opin. Cell Biol. 39, 84-92 (2016).

3. Y. Omori et al., Identification of G protein-coupled receptors (GPCRs) in primary cilia and their possible involvement in body weight control. PLoS One 10, e0128422 (2015)

4. D. Jin et al., Prostaglandin signalling regulates ciliogenesis by modulating intraflagellar transport. Nat. Cell Biol. 16, 841-851 (2014).

5. J. S. Domire et al., Dopamine receptor 1 localizes to neuronal cilia in a dynamic process that requires the Bardet-Biedl syndrome proteins. Cell. Mol. Life Sci. 68, 29512960 (2011).

6. E. S. Mitchell, J. F. Neumaier, 5-HT6 receptors: A novel target for cognitive enhancement. Pharmacol. Ther. 108, 320-333 (2005).

7. I. Brailov et al., Localization of 5-HT(6) receptors at the plasma membrane of neuronal cilia in the rat brain. Brain Res. 872, 271-275 (2000).

8. S. Su et al., Genetically encoded calcium indicator illuminates calcium dynamics in primary cilia. Nat. Methods 10, 1105-1107 (2013).

9. L. Hu, B. Wang, Y. Zhang, Serotonin 5-HT6 receptors affect cognition in a mouse model of Alzheimer's disease by regulating cilia function. Alzheimers Res. Ther. 9, 76 (2017).

10. D. U. Mick et al., Proteomics of primary cilia by proximity labeling. Dev. Cell 35, 497512 (2015).

11. Y. H. Choi et al., Polycystin-2 and phosphodiesterase $4 \mathrm{C}$ are components of a ciliary Akinase anchoring protein complex that is disrupted in cystic kidney diseases. Proc Natl. Acad. Sci. U.S.A. 108, 10679-10684 (2011).

12. X. He et al., The G protein $\alpha$ subunit Gas is a tumor suppressor in sonic hedgehogdriven medulloblastoma. Nat. Med. 20, 1035-1042 (2014).

13. S. M. Guadiana et al., Type 3 adenylyl cyclase and somatostatin receptor 3 expression persists in aged rat neocortical and hippocampal neuronal cilia. Front. Aging Neurosci. 8, 127 (2016).

14. V. A. Bachmann et al., Gpr161 anchoring of PKA consolidates GPCR and CAMP sig naling. Proc. Natl. Acad. Sci. U.S.A. 113, 7786-7791 (2016).

15. L. Vuolo, A. Herrera, B. Torroba, A. Menendez, S. Pons, Ciliary adenylyl cyclases control the hedgehog pathway. J. Cell Sci. 128, 2928-2937 (2015).

16. R. Y. Kwon, S. Temiyasathit, P. Tummala, C. C. Quah, C. R. Jacobs, Primary ciliumdependent mechanosensing is mediated by adenylyl cyclase 6 and cyclic AMP in bone cells. FASEB J. 24, 2859-2868 (2010).

17. S. Mukhopadhyay et al., The ciliary G-protein-coupled receptor Gpr161 negatively regulates the sonic hedgehog pathway via CAMP signaling. Cell 152, 210-223 (2013)

18. S. Mukhopadhyay, R. Rohatgi, G-protein-coupled receptors, hedgehog signaling and primary cilia. Semin. Cell Dev. Biol. 33, 63-72 (2014).

19. B. S. Moore et al., Cilia have high CAMP levels that are inhibited by sonic hedgehog regulated calcium dynamics. Proc. Natl. Acad. Sci. U.S.A. 113, 13069-13074 (2016).

20. F. Bangs, K. V. Anderson, Primary cilia and mammalian hedgehog signaling. Cold Spring Harb. Perspect. Biol. 9, a028175 (2017).

21. M. Barzi, J. Berenguer, A. Menendez, R. Alvarez-Rodriguez, S. Pons, Sonic-hedgehogmediated proliferation requires the localization of PKA to the cilium base. J. Cell Sci. 123, 62-69 (2010)

22. A. V. Loktev, P. K. Jackson, Neuropeptide $Y$ family receptors traffic via the BardetBiedl syndrome pathway to signal in neuronal primary cilia. Cell Rep. 5, 1316-1329 (2013).

23. Q. Wang et al., Adenylyl cyclase 5 deficiency reduces renal cyclic AMP and cyst growth in an orthologous mouse model of polycystic kidney disease. Kidney Int. 93, 403-415 (2018).

24. S. Mukherjee et al., A novel biosensor to study cAMP dynamics in cilia and flagella. elife 5, e14052 (2016).

25. A. Marley, R. W. Choy, M. von Zastrow, GPR88 reveals a discrete function of primary cilia as selective insulators of GPCR cross-talk. PLoS One 8, e70857 (2013).

26. J. Klarenbeek, J. Goedhart, A. van Batenburg, D. Groenewald, K. Jalink, Fourthgeneration epac-based FRET sensors for cAMP feature exceptional brightness, photostability and dynamic range: Characterization of dedicated sensors for FLIM, for ratiometry and with high affinity. PLoS One 10, e0122513 (2015).

27. N. A. Duldulao, S. Lee, Z. Sun, Cilia localization is essential for in vivo functions of the Joubert syndrome protein Arl13b/Scorpion. Development 136, 4033-4042 (2009).

28. R. Kohen, L. A. Fashingbauer, D. E. Heidmann, C. R. Guthrie, M. W. Hamblin, Cloning of the mouse 5-HT6 serotonin receptor and mutagenesis studies of the third cytoplasmic loop. Brain Res. Mol. Brain Res. 90, 110-117 (2001). and a $480 \mathrm{~nm} / 535 \mathrm{~nm}$ emission ratio $(35,56)$. Resting CAMP in cilia and cell bodies was determined by careful calibration of targeted and untargeted CAMP sensors in digitonin permeabilized cells.

ACKNOWLEDGMENTS. We thank Madelaine Ma, Maria Ting, and Meeru Kumar for helping with experiments. We are grateful to Takanari Inoue, Mark von Zastrow, Kees Jalink, Richard Bouley, and Carole A. Parent for providing plasmids and all the researchers who kindly deposited the remaining plasmids used for this study in Addgene. This work was generously supported by grants to A.M.H. from NIH/National Institutes for Dental and Craniofacial Research (R21 DE025921), Harvard Catalyst (NIH 1UI1 TR00102-01), and the Department of Veterans Affairs (I21 BX004093). The VA Boston Healthcare System confocal microscopy core was graciously funded by The Department of Veterans Affairs (1IS1BX003538-01 and 1IS1BX004786-01).

29. J. E. Siljee et al., Subcellular localization of MC4R with $A D C Y 3$ at neuronal primary cilia underlies a common pathway for genetic predisposition to obesity. Nat. Genet. 50, 180-185 (2018).

30. D. K. Breslow, E. F. Koslover, F. Seydel, A. J. Spakowitz, M. V. Nachury, An in vitro assay for entry into cilia reveals unique properties of the soluble diffusion barrier. $J$. Cell Biol. 203, 129-147 (2013)

31. D. K. Breslow, M. V. Nachury, Analysis of soluble protein entry into primary cilia using semipermeabilized cells. Methods Cell Biol. 127, 203-221 (2015).

32. M. K. Raychowdhury et al., Vasopressin receptor-mediated functional signaling pathway in primary cilia of renal epithelial cells. Am. J. Physiol. Renal. Physiol. 296, F87-F97 (2009).

33. R. Bouley et al., Functional role of the NPxxY motif in internalization of the type 2 vasopressin receptor in LLC-PK1 cells. Am. J. Physiol. Cell Physiol. 285, C750-C762 (2003).

34. T. N. Feinstein et al., Noncanonical control of vasopressin receptor type 2 signaling by retromer and arrestin. J. Biol. Chem. 288, 27849-27860 (2013).

35. K. Lefkimmiatis, M. P. Moyer, S. Curci, A. M. Hofer, "cAMP sponge": A buffer for cyclic adenosine 3', 5'-monophosphate. PLoS One 4, e7649 (2009).

36. L. Liu, S. Das, W. Losert, C. A. Parent, mTORC2 regulates neutrophil chemotaxis in a CAMP- and RhoA-dependent fashion. Dev. Cell 19, 845-857 (2010).

37. T. Y. Besschetnova et al., Identification of signaling pathways regulating primary cilium length and flow-mediated adaptation. Curr. Biol. 20, 182-187 (2010).

38. P. G. DeCaen, M. Delling, T. N. Vien, D. E. Clapham, Direct recording and molecular identification of the calcium channel of primary cilia. Nature 504, 315-318 (2013).

39. M. Delling, P. G. DeCaen, J. F. Doerner, S. Febvay, D. E. Clapham, Primary cilia are specialized calcium signalling organelles. Nature 504, 311-314 (2013).

40. J. K. Chen, J. Taipale, K. E. Young, T. Maiti, P. A. Beachy, Small molecule modulation of smoothened activity. Proc. Natl. Acad. Sci. U.S.A. 99, 14071-14076 (2002).

41. K. Pal, S. Mukhopadhyay, Primary cilium and sonic hedgehog signaling during neural tube patterning: Role of GPCRs and second messengers. Dev. Neurobiol. 75, 337-348 (2014).

42. K. K. Chahal, M. Parle, R. Abagyan, Hedgehog pathway and smoothened inhibitors in cancer therapies. Anticancer Drugs 29, 387-401 (2018)

43. J. Taipale, P. A. Beachy, The hedgehog and Wnt signalling pathways in cancer. Nature 411, 349-354 (2001).

44. S. M. Guadiana et al., Arborization of dendrites by developing neocortical neurons is dependent on primary cilia and type 3 adenylyl cyclase. J. Neurosci. 33, 2626-2638 (2013).

45. Y. Tian, B. Peng, X. Fu, New ADCY3 variants dance in obesity etiology. Trends Endocrinol. Metab. 29, 361-363 (2018).

46. E. Y. J. Park, M. Kwak, K. Ha, I. So, Identification of clustered phosphorylation sites in PKD2L1: How PKD2L1 channel activation is regulated by cyclic adenosine monophosphate signaling pathway. Pflugers Arch. 470, 505-516 (2018).

47. S. J. Kleene, N. K. Kleene, The native TRPP2-dependent channel of murine renal primary cilia. Am. J. Physiol. Renal. Physiol. 312, F96-F108 (2017).

48. C. C. Ruppersburg, H. C. Hartzell, The Ca2+-activated Cl- channel ANO1/TMEM16A regulates primary ciliogenesis. Mol. Biol. Cell 25, 1793-1807 (2014).

49. S. C. Phua, Y. C. Lin, T. Inoue, An intelligent nano-antenna: Primary cilium harnesses TRP channels to decode polymodal stimuli. Cell Calcium 58, 415-422 (2015).

50. X. Liu et al., Polycystin-2 is an essential ion channel subunit in the primary cilium of the renal collecting duct epithelium. elife 7, e33183 (2018).

51. M. Brodsky et al., $5-\mathrm{HT}_{6}$ receptor blockade regulates primary cilia morphology in striatal neurons. Brain Res. 1660, 10-19 (2017).

52. R. Khoury, N. Grysman, J. Gold, K. Patel, G. T. Grossberg, The role of 5 HT6-receptor antagonists in Alzheimer's disease: An update. Expert Opin. Investig. Drugs 27, 523533 (2018).

53. A. I. Masyuk et al., Ciliary subcellular localization of TGR5 determines the cholangiocyte functional response to bile acid signaling. Am. J. Physiol. Gastrointest. Liver Physiol. 304, G1013-G1024 (2013).

54. S. C. Phua, Y. Nihongaki, T. Inoue, Autonomy declared by primary cilia through compartmentalization of membrane phosphoinositides. Curr. Opin. Cell Biol. 50, 7278 (2018).

55. G. V. Pusapati et al., G protein-coupled receptors control the sensitivity of cells to the morphogen sonic hedgehog. Sci. Signal. 11, eaao5749 (2018).

56. K. Lefkimmiatis, D. Leronni, A. M. Hofer, The inner and outer compartments of mitochondria are sites of distinct CAMP/PKA signaling dynamics. J. Cell Biol. 202, 453462 (2013). 